\title{
Adaptive Chattering-Free Sliding Mode Control of Chaotic Systems with Unknown Input Nonlinearity via Smooth Hyperbolic Tangent Function
}

\author{
Jiunn-Shiou Fang $\mathbb{D}^{1},{ }^{1}$ Jason Sheng-Hong Tsai $\mathbb{C}^{1},{ }^{1}$ Jun-Juh Yan $\mathbb{D}^{2},{ }^{2}$ and Shu-Mei Guo $\mathbb{D}^{3}$ \\ ${ }^{1}$ Department of Electrical Engineering, National Cheng-Kung University, Tainan 701, Taiwan \\ ${ }^{2}$ Department of Electronic Engineering, National Chin-Yi University of Technology, Taichung 41107, Taiwan \\ ${ }^{3}$ Department of Computer Science and Information Engineering, National Cheng-Kung University, Tainan 701, Taiwan
}

Correspondence should be addressed to Jason Sheng-Hong Tsai; shtsai@mail.ncku.edu.tw and Jun-Juh Yan; jjyan@stu.edu.tw

Received 17 May 2019; Revised 17 July 2019; Accepted 1 August 2019; Published 7 October 2019

Academic Editor: Alessandro Lo Schiavo

Copyright (c) 2019 Jiunn-Shiou Fang et al. This is an open access article distributed under the Creative Commons Attribution License, which permits unrestricted use, distribution, and reproduction in any medium, provided the original work is properly cited.

\begin{abstract}
The design of adaptive chattering-free sliding mode controller (SMC) for chaotic systems with unknown input nonlinearities is studied in this paper. A smooth hyperbolic tangent function is utilized to replace the discontinuous sign function; therefore, the proposed adaptive SMC ensures that not only the chaos phenomenon can be suppressed effectively but also the chattering often appearing in the traditional discontinuous SMC with sign function is eliminated, even when the unknown input nonlinearity is present. A sufficient condition for stability of closed-loop system is acquired by Lyapunov theory. The numerical simulation results are illustrated to verify the proposed adaptive sliding mode control method.
\end{abstract}

\section{Introduction}

In recent decades, sliding mode control is one of the popular control theories due to its robustness and insensitivity to parameter uncertainty and external disturbance $[1,2]$. The trajectories of SMC systems can be driven onto a specified sliding surface and slide toward desired stable condition, which is called sliding motion. There are many methodologies used to select sliding surfaces, while the different control law is obtained, and it is guaranteed that the controlled system is stable [2]. In general, the SMC and switched system control strategies are usually adopted to the practical application. For example, in $[3,4]$, the new SMC-based fuzzy controller and robust hybrid controller are applied to the 7-degree-of-freedom upper-limb exoskeleton robot such that the undesired external disturbance can be suppressed. Therefore, the SMC can be used to overcome undesired external disturbance successfully; in [5], a class of linear switched system is discussed for the cooperative stabilization problem and a novel class of switching signals is proposed. Based on above descriptions, the SMC is adopted to cope with input nonlinearity considered in this paper due to its robustness.

In traditional SMC, the control force is difficult to achieve, which has high frequency chattering phenomenon due to the discontinuous sign function. Therefore, many quasisliding mode control techniques have been proposed $[2,6-8]$, and the chattering phenomenon is eliminated due to the controller designed by using a continuous function. On the other hand, chaotic systems have been proven the existence which is extensively and become an interesting topic in physical systems [9-11]. Also, the chaotic systems have special nonlinear dynamic behavior [7, 12]. In general, there are two sides to such physical systems [11]; the first side is the beneficial feature, such as chemical reactions and encrypted communication systems. On the other side, the chaos phenomenon in some engineering systems is highly unexpected for its applications, such as electronic systems, power converters, and high-precision mechanical systems. Therefore, many different methods have been proposed to solve the problems of chaos control and suppression for chaotic systems, such as adaptive feedback control [13-16], 
sliding mode control [11, 17, 18], PID control [19-21], optimal control [22-26], and robust control [27-29] among many others $[30,31]$.

As described above, many modified methodologies have been developed to overcome the chattering phenomenon in traditional SMC, but they have not been well discussed when the system structure is subjected to input nonlinearity and even the input nonlinearity is unknown. It is well known that in practical systems, unavoidable external nonlinear perturbations do exist in control input, which not only affect system stability but also decrease the input performance and response. Furthermore, since the feature of chaotic system is very sensitive to parameter uncertainty and external perturbation [11], the stability analysis of controlled system with input nonlinearity is an important issue for chaotic systems. Recently, some papers $[11,32,33]$ have researched SMC design for the chaotic systems with nonlinear input, but they only discussed the control design when input nonlinearity is well known. $\mathrm{Hu}$ et al. [34] proposed a SMC-based adaptive controller to deal with nonlinear input, but the adaptive law is high order. However, we present a low-order adaptive law to omit the harmful effects of nonlinear input. To our knowledge, the chattering-free SMC design with unknown input nonlinearity is still not well discussed.

In this paper, the adaptive continuous SMC design for chaotic systems with unknown input nonlinearity is studied. The main contribution is to introduce a new technique of adaptive sliding mode control with a smooth hyperbolic tangent function to avoid chattering. In addition, the undesired chaotic behavior of the considered chaotic systems can be fully suppressed even with unknown input nonlinearities. Finally, we present numerical simulation results to illustrate the effectiveness of the proposed adaptive continuous SMC scheme.

1.1. Notations. In this paper, $R^{n}$ represents the $n$-dimensional Euclidean space, $R^{n \times m}$ denotes the set of all real $n$ by $m$ matrices, the superscript "+" denotes the matrix generalized inverse, and $I_{m}$ stands for $m$ by $m$ identity matrices. $\|W\|$ denotes the Euclidean norm, when $W$ is a vector, and the induced norm, when $W$ is a matrix. $\lambda_{\max }(W)$ denotes the maximum eigenvalue of matrix $W$. $|W|$ represents the absolute value of $W$, and $\operatorname{sign}(s)$ is the sign function of $s$; if $s>0$, $\operatorname{sign}(s)=1$; if $s=0$, $\operatorname{sign}(s)=0$; and if $s<0, \operatorname{sign}(s)=-1$.

\section{System Description and Problem Formulation}

Consider a general class of chaotic systems given below:

$$
\dot{x}(t)=A x+f(x, t),
$$

where $A \in R^{n \times n}$ is the system matrix and $x \in R^{n}$ is the system state vector. $f(x, t) \in R^{n \times 1}$ denotes the nonlinear vector of systems. Without loss of generality, we make the following assumption about system (1).
Assumption 1. The dynamic system (1) can be written as

$$
\dot{x}(t)=A x+B g(x, t),
$$

where $B \in R^{n \times m}$ and $g(x, t) \in R^{m \times 1}$. The pair $A, B$ is controllable.

Remark 1. Assumption 1 is not restrictive. Many nonlinear chaotic systems described by (2) can be found. For example, the Matsumoto-Chua-Kobayashi circuit, Rössler system, modified Chua's circuit, Lorenz system, Duffing-Holmes system, Lü system, and Chen chaotic dynamical system.

To suppress the chaos oscillation behavior, we introduce a control vector subjected to unknown nonlinearity described as

$$
\dot{x}(t)=A x+B g(x, t)+B \varphi(u(t))
$$

where $\varphi(u(t))=\left[\varphi_{1}\left(u_{1}\right) \cdots \varphi_{m}\left(u_{m}\right)\right]^{T} \in R^{m \times 1}$ is the control vector with nonlinearity. The continuous nonlinear function satisfies $\varphi(0)=0$, where $\phi_{i}\left(u_{i}(t)\right) \in R \longrightarrow R$ with the law $u_{i}(t) \longrightarrow \varphi_{i}\left(u_{i}(t)\right)$, satisfying

$$
\beta_{2, i} u_{i}^{2} \geq u_{i} \varphi_{i}\left(u_{i}\right) \geq \beta_{1, i} u_{i}^{2}
$$

where $\beta_{1 i}$ and $\beta_{2 i}$ are unknown positive nonzero constants but bounded. Figure 1 shows a nonlinear function $\varphi_{i}\left(u_{i}(t)\right)$ inside the sectors $\beta_{1 i}$ and $\beta_{2 i}$.

In SMC, the traditional controller is difficult to implement because there is an important adverse problem of highfrequency chattering phenomenon. In order to improve this issue, the following lemma with smooth hyperbolic tangent function is introduced.

Lemma 1. There always exists a constant $\gamma_{i}>0$ for all $s_{i} \neq 0$ such that

$$
s_{i} \cdot \tanh \left(s_{i}\right)>\gamma_{i} s_{i} \cdot \operatorname{sign}\left(s_{i}\right)
$$

Proof. Since $\tanh \left(s_{i}\right)=\left(e^{j s_{i}}-e^{-j s_{i}}\right) /\left(e^{j s_{i}}+e^{-j s_{i}}\right), j>0$, obviously, $s_{i} \cdot \tanh \left(s_{i}\right)>0$ and $s_{i} \cdot \operatorname{sign}\left(s_{i}\right)=\left|s_{i}\right|>0$ for all $s_{i} \neq 0$. In general, there always exists a positive constant $\varepsilon_{i} \in(0,1)$ satisfying

$$
s_{i} \operatorname{sign}\left(s_{i}\right)<s_{i} \tanh \left(s_{i}\right)+\varepsilon_{i} s_{i} \operatorname{sign}\left(s_{i}\right) .
$$

Also, it can be rewritten as

$$
s_{i} \tanh \left(s_{i}\right)>\left(1-\varepsilon_{i}\right) s_{i} \operatorname{sign}\left(s_{i}\right)=\gamma_{i} s_{i} \operatorname{sign}\left(s_{i}\right),
$$

where $\gamma_{i}=\left(1-\varepsilon_{i}\right)$. Therefore, there always exists a constant $\gamma_{i}>0$ satisfying $s_{i} \cdot \tanh \left(s_{i}\right)>\gamma_{i} s_{i} \cdot \operatorname{sign}\left(s_{i}\right)$ for all $s_{i} \neq 0$.

It is worthy to mention that the existing but unknown parameter $\gamma_{i}$ does not appear in our proposed controller due to the adaptive control approach. By Lemma 1, a smooth continuous switching function is used to construct our control design such that the chattering phenomenon can be improved. As shown in Figure 2, the different parameters $j$ are discussed for hyperbolic tangent function. 


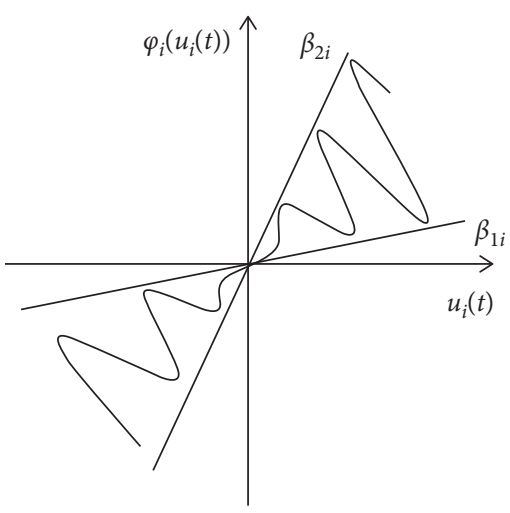

FIgURe 1: The scalar nonlinear function $\varphi_{i}\left(u_{i}(t)\right)$.

According to Figure 2, the hyperbolic tangent function with a large value of $j$ is close to the discontinuous sign function while the chattering phenomenon might appear. Therefore, the parameter $j$ should be assigned by an appropriate value for avoiding the undesired chattering in SMC.

\section{The Control Design Algorithm}

In consequence, to complete the control objective mentioned above, there are two major steps. First, one needs to design an appropriate switching surface for the control system with input nonlinearity such that the stability of the dynamics on the sliding manifold defined later can be ensured. Second, one needs to propose a continuous adaptive SMC such that the existence of the sliding motion can be guaranteed without chattering.

First, the proportional-integral (PI) type sliding surface is first defined as follows:

$$
S(t)=C x(t)-\int_{0}^{t}(C A+K) x(\tau) d \tau,
$$

where $S(t) \in R^{m}, C \in R^{m \times n}$, and $K \in R^{m \times n}$ and matrix $C=$ $B^{+}=\left(B^{T} B\right)^{-1} B^{T}$ results in $C B=I_{m} . B^{+}$is the generalized inverse of $B$, and $B$ is the full-column rank. $I_{m} \in R^{m}$ is the identity matrix. The control matrix $K$ satisfies $\lambda_{\max }$ $(A+B K)<0$. As long as the system can operate in the sliding mode, the controlled dynamics will satisfy the following equation:

$$
\begin{aligned}
& \dot{S}(t)=0, \\
& S(t)=0 .
\end{aligned}
$$

Therefore, when the system operates in the sliding mode, we can obtain the equivalent control $\varphi_{\text {eq }}(u(t))$ by differentiating (8) with respect to time and substituting from (3):

$$
\dot{S}(t)=-K x(t)+g(x, t)+\varphi_{\mathrm{eq}}(u(t))=0,
$$

where $C B=B^{+} B=I_{m}$ has been introduced. Obviously, the equivalent control $\varphi_{\mathrm{eq}}(u(t))$ in the sliding mode is obtained by

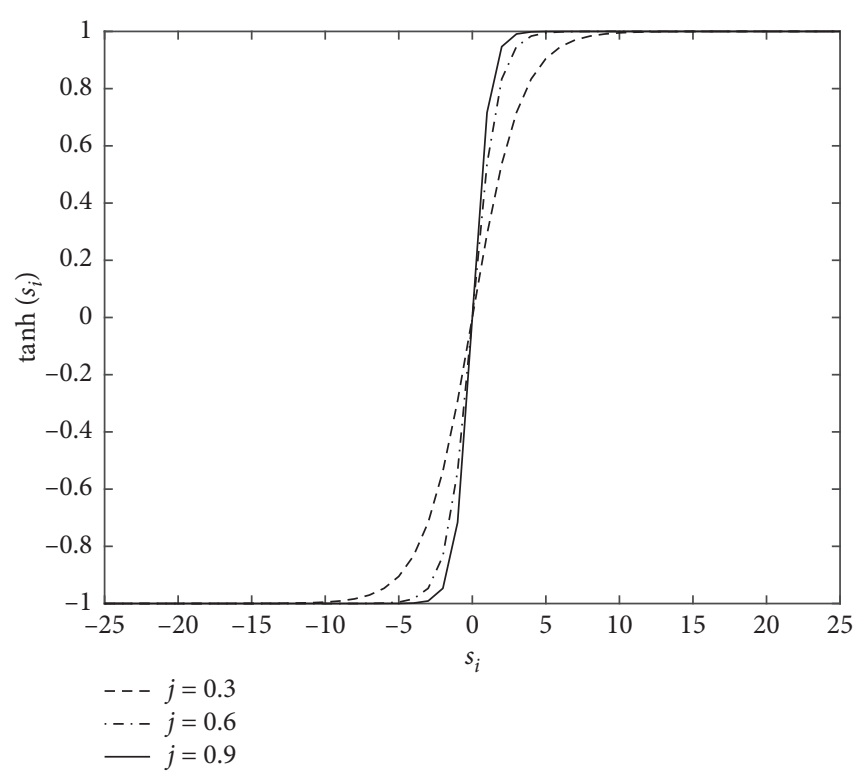

FIgURE 2: The $\tanh \left(s_{i}\right)=\left(e^{j s_{i}}-e^{-j s_{i}}\right) /\left(e^{j s_{i}}+e^{-j s_{i}}\right)$ function with different $j$.

$$
\varphi_{\mathrm{eq}}(u(t))=K x(t)-g(x, t) .
$$

Substituting (11) into (3), we have

$$
\dot{x}(t)=(A+B K) x(t) .
$$

According the above discussion and (12), we can conclude that when the system is in the sliding manifold, the controlled chaotic system in the sliding mode is stable if matrix $K$ satisfies $\lambda_{\max }(A+B K)<0$. Now, to guarantee the existence of the sliding mode, the continuous sliding controller and adaptive law are proposed, respectively, as

$$
\begin{aligned}
& u(t)=-\alpha \widehat{\eta}(t)(\|g(x, t)-K x\|+\delta) \tanh (S), \\
& \dot{\hat{\eta}}(t)=\alpha(\|g(x, t)-K x\|+\delta) \cdot\|S\| .
\end{aligned}
$$

From (13) and (14), we have

$$
\begin{aligned}
u_{i}(t) & =-\alpha \widehat{\eta}(t)(\|g(x, t)-K x\|+\delta) \tanh \left(s_{i}\right), \\
\dot{\hat{\eta}}(t) & =\alpha(\|g(x, t)-K x\|+\delta) \cdot\|S\|,
\end{aligned}
$$

where $\alpha>1, \delta>0$ are constants and can be assigned. The factor $\hat{\eta}(t)$ is used to estimate the unknown input nonlinearity. The control block diagram is shown in Figure 3.

Theorem 1. Consider dynamic system (3) with unknown input nonlinearity. If the continuous adaptive SMC is properly designed as (13) and (14), then the system trajectory will be controlled to the sliding surface $S(t)=0$ even with unknown input nonlinearities.

Proof. Let us consider a Lyapunov function for a closed-loop system as follows:

$$
V(t)=\frac{1}{2}\left(S^{T} S+\eta e^{2}\right)
$$




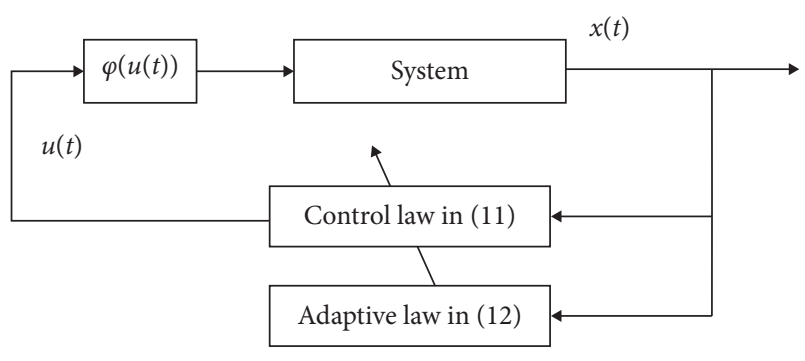

Figure 3: The control block diagram.

where $\eta=\gamma_{1} \cdot \beta_{1}, \beta_{1}=\min _{i \in[1, m]} \beta_{1, i}, \gamma_{1}=\min _{i \in[1, m]} \gamma_{i}, e=$ $\hat{\eta}(t)-\eta^{-1}$, and $\dot{e}=\dot{\hat{\eta}}(t)$ and $\eta$ is an unknown constant. Then, the Lyapunov function (16) derivative with respect to time is obtained as

$$
\begin{aligned}
\dot{V}(t) & =S^{T} \dot{S}+e \eta \dot{e}=S^{T}(g(x, t)-K x+\varphi(u))+e \eta \dot{e} \\
& \leq\|S\| \cdot(\|g(x, t)-K x\|+\delta)+S^{T} \varphi(u)+e \eta \dot{e} .
\end{aligned}
$$

Since $\beta_{2, i} u_{i}^{2} \geq u_{i} \varphi_{i}\left(u_{i}\right) \geq \beta_{1, i} u_{i}^{2}$, one has

$$
\begin{aligned}
& -\alpha \widehat{\eta}(t)(\|g(x, t)-K x\|+\delta) \tanh \left(s_{i}\right) \varphi\left(u_{i}\right) \\
& \quad \geq \beta_{1, i} \alpha^{2}(\widehat{\eta}(t))^{2}(\|g(x, t)-K x\|+\delta)^{2} \tanh ^{2}\left(s_{i}\right),
\end{aligned}
$$

and then

$$
-\tanh \left(s_{i}\right) \varphi\left(u_{i}\right) \geq \alpha \beta_{1, i} \widehat{\eta}(t)(\|g(x, t)-K x\|+\delta) \tanh ^{2}\left(s_{i}\right) .
$$

Since $s_{i}^{2} \geq 0, \gamma_{1}=\min _{i \in[1, m]} \gamma_{i}, \beta_{1}=\min _{i \in[1, m]} \beta_{1, i}$, and

$$
s_{i} \cdot \tanh \left(s_{i}\right)>\gamma_{i} s_{i} \cdot \operatorname{sign}\left(s_{i}\right), \quad \text { for all } s_{i} \neq 0,
$$

by substituting (20) into (19), we get the following result:

$$
\begin{aligned}
-\tanh \left(s_{i}\right) s_{i}^{2} \varphi\left(u_{i}\right) & \geq \alpha \beta_{1, i} \widehat{\eta}(t)(\|g(x, t)-K x\|+\delta) s_{i}^{2} \tanh ^{2}\left(s_{i}\right), \\
-s_{i} \varphi\left(u_{i}\right) & \geq \alpha \beta_{1, i} \widehat{\eta}(t)(\|g(x, t)-K x\|+\delta) s_{i} \tanh \left(s_{i}\right), \\
-s_{i} \varphi\left(u_{i}\right) & \geq \alpha \gamma_{i} \beta_{1, i} \widehat{\eta}(t)(\|g(x, t)-K x\|+\delta) s_{i} \cdot \operatorname{sign}\left(s_{i}\right), \\
-s_{i} \varphi\left(u_{i}\right) & \geq \alpha \gamma_{1} \beta_{1} \widehat{\eta}(t)(\|g(x, t)-K x\|+\delta) s_{i} \cdot \operatorname{sign}\left(s_{i}\right) .
\end{aligned}
$$

Let $\eta=\beta_{1} \gamma_{1}$ and $\sum_{i=1}^{m}\left|s_{i}\right| \geq\|S\|$; then, we have

$$
-s_{i} \varphi\left(u_{i}\right) \geq \alpha \eta \widehat{\eta}(t)(\|g(x, t)-K x\|+\delta) \cdot\left|s_{i}\right|,
$$

thus

$$
\begin{aligned}
-\sum_{i=1}^{m} s_{i}\left(\varphi u_{i}\right) & \geq \alpha \eta \widehat{\eta}(t)(\|g(x, t)-K x\|+\delta) \cdot \sum_{i=1}^{m}\left|s_{i}\right| \\
& \geq \alpha \eta \widehat{\eta}(t)(\|g(x, t)-K x\|+\delta) \cdot\|S\| .
\end{aligned}
$$

Furthermore, $\sum_{i=1}^{m} s_{i} \varphi\left(u_{i}\right)=S^{T} \varphi(u)$, and one has

$$
S^{T} \varphi(u) \leq-\alpha \eta \widehat{\eta}(t)(\|g(x, t)-K x\|+\delta) \cdot\|S\| .
$$

By substituting (13), (14), and (24) into the derivative of Lyapunov function (16), one has

$$
\begin{aligned}
\dot{V}(t) \leq & \|S\| \cdot(\|g(x, t)-K x\|+\delta) \\
& -\alpha \eta \widehat{\eta}(t)(\|g(x, t)-K x\|+\delta) \cdot\|S\|+e \eta \dot{e} \\
= & (1-\alpha)\|S\| \cdot(\|g(x, t)-K x\|+\delta) \\
& -\alpha\left(\widehat{\eta}(t)-\eta^{-1}\right) \eta(\|g(x, t)-K x\|+\delta) \cdot\|S\|+e \eta \dot{\hat{\eta}}(t) \\
= & (1-\alpha)(\|g(x, t)-K x\|+\delta) \cdot\|S\| .
\end{aligned}
$$

Let $w(t)=(1-\alpha)(\|g(x, t)-K x\|+\delta) \cdot\|S\|$; then, one has

$$
\begin{gathered}
V(t) \leq V(0)-\int_{0}^{t} w(\lambda) d \lambda \\
\Longrightarrow V(0) \geq V(t)+\int_{0}^{t} w(\lambda) d \lambda \geq \int_{0}^{t} w(\lambda) d \lambda .
\end{gathered}
$$

Thus, by using Barbalat lemma [31], we obtain $\lim _{t \rightarrow \infty} w(t)=0$. Furthermore, since $\alpha>1,\|g(x, t)-K x\|$ $+\delta>0, S \longrightarrow 0$ as $t \longrightarrow \infty$. Hence, the proof is achieved completely.

Remark 2. Since a continuous adaptive SMC with a smooth hyperbolic tangent function is obtained, there is no highfrequency switching operation in sliding mode controller and the chattering is removed.

\section{Numerical Simulations}

In this section, to verify the proposed controller, we give two illustrative examples.

Example 1. Consider modified Chua's circuit, and the dynamic system can be described as [35]

$$
\begin{aligned}
& \dot{x}_{1}(t)=p x_{2}(t)+\frac{p}{7} x_{1}(t)+f\left(x_{1}, t\right), \\
& \dot{x}_{2}(t)=x_{1}(t)-x_{2}(t)+x_{3}(t), \\
& \dot{x}_{3}(t)=-q x_{2}(t),
\end{aligned}
$$

with the nonlinear function

$$
f\left(x_{1}, t\right)=-2 p m_{0} x_{1}^{3}
$$

where $p>0, q>0, m_{0}$ are the system parameters and $x(t)=\left[\begin{array}{lll}x_{1} & x_{2} & x_{3}\end{array}\right]^{T}$ is the state vector. The chaos response of Chua's system without control force is shown in Figure 4. It is easy to verify that system (27) with nonlinear control input $\phi(u(t))$ can be represented as

$$
\dot{x}=A x+B(g(x, t)+\phi(u(t)))
$$

where $A=\left[\begin{array}{ccc}p / 7 & p & 0 \\ 1 & -1 & 1 \\ 0 & -q & 0\end{array}\right], \quad B=\left[\begin{array}{l}1 \\ 0 \\ 0\end{array}\right], \quad g(x)=\left[-2 p m_{0} x_{1}^{3}\right]$, $p=10, q=100 / 7$, and $m_{0}=1 / 7$.

The initial state condition is given by $x_{0}=$ $\left[\begin{array}{lll}-1.2 & 0.2 & -2\end{array}\right]^{T}$. The controller parameters are given by 


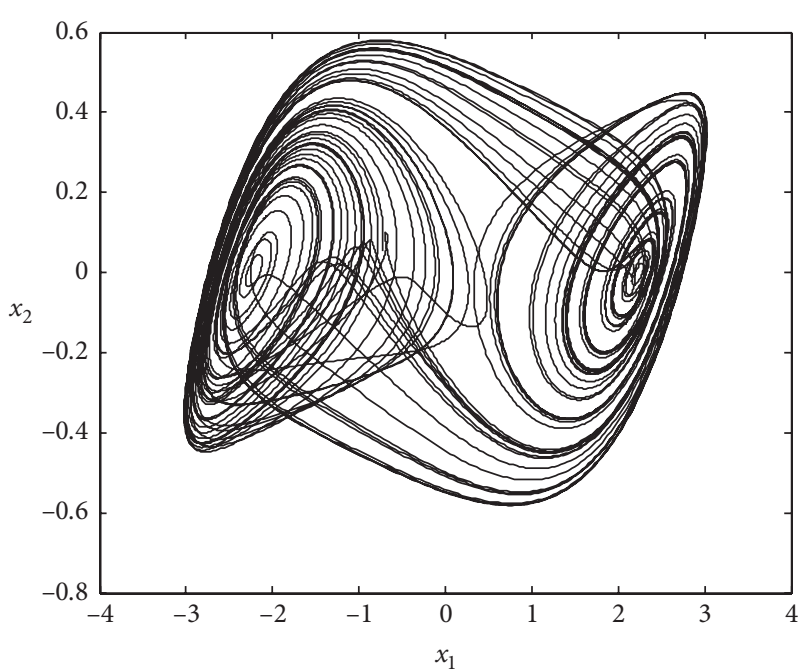

FIGURE 4: The chaos response of modified Chua's circuit without control force.

$j=1, \quad \delta=8, \quad \alpha=1.2, \quad$ and $\quad \hat{\eta}_{0}=0 . \quad$ Let $K=\left[\begin{array}{lll}-6.4286 & -1.7143-4.58\end{array}\right]$ such that the eigenvalues of the system are placed on $\{-2,-3,-1\}$. Also, for simulation, the unknown nonlinear function is given as

$$
\varphi(u(t))=(1+0.3 \sin (u(t))) u(t) .
$$

Under the above-mentioned input nonlinearity, the corresponding state responses, the proposed controller (13), the sliding surface (8), and adaptive parameter are shown in Figures 5-8, respectively. In order to compare the proposed approach with the traditional SMC, the tanh function is replaced by the traditional sign function in the controller (13). Based on traditional SMC, the corresponding state responses, the control input, the sliding surface, and adaptive parameter are shown in Figures 9-12, respectively. Comparing Figures 5-8 with Figures 9-12, it is shown that the undesired chattering phenomenon can be fully suppressed by the proposed adaptive chattering-free control law (13).

Example 2. Consider the chaotic Lorenz system [36] described as

$$
\begin{aligned}
& \dot{x}_{1}(t)=-m_{1} x_{1}(t)+m_{1} x_{2}(t), \\
& \dot{x}_{2}(t)=m_{2} x_{1}(t)-x_{2}(t)+f_{1}(x, t), \\
& \dot{x}_{3}(t)=-m_{3} x_{2}(t)+f_{2}(x, t),
\end{aligned}
$$

where $m_{i}, i=1,2,3$ are the system parameters and $x(t)=$ $\left[\begin{array}{lll}x_{1} & x_{2} & x_{3}\end{array}\right]^{T}$ are the system states. The chaos response of the Lorenz system without control force is shown in Figure 13. Also, the state space with control input $\phi(u(t))$ can be rewritten as

$$
\dot{x}=A x+B(g(x, t)+\phi(u(t)))
$$

where $\quad A=\left[\begin{array}{ccc}-m_{1} & m_{1} & 0 \\ m_{2} & -1 & 0 \\ 0 & 0 & -m_{3}\end{array}\right], \quad B=\left[\begin{array}{ll}0 & 0 \\ 1 & 0 \\ 0 & 1\end{array}\right], \quad g(x, t)=$ $\left[f_{1}(x, t) f_{2}(x, t)\right]^{T}=\left[\begin{array}{ll}-x_{1}(t) x_{3}(t) & x_{1}(t) x_{2}(t)\end{array}\right]^{T}, m_{1}=10$, $m_{2}=28$, and $m_{3}=8 / 3$.

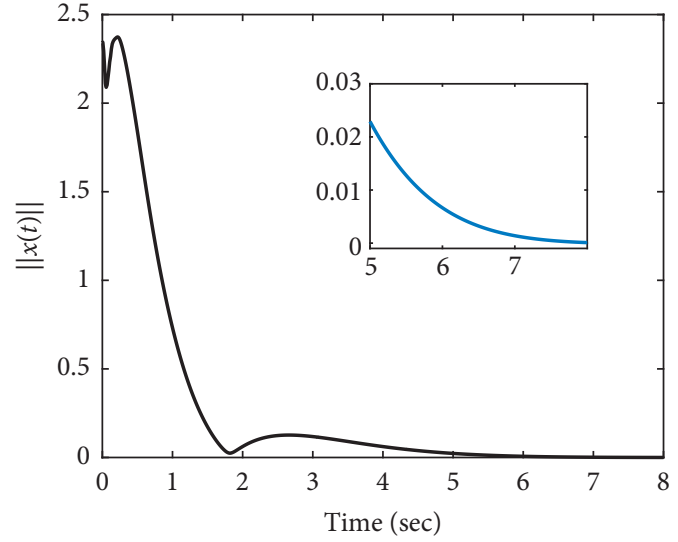

Figure 5: The system state response with the proposed continuous controller.

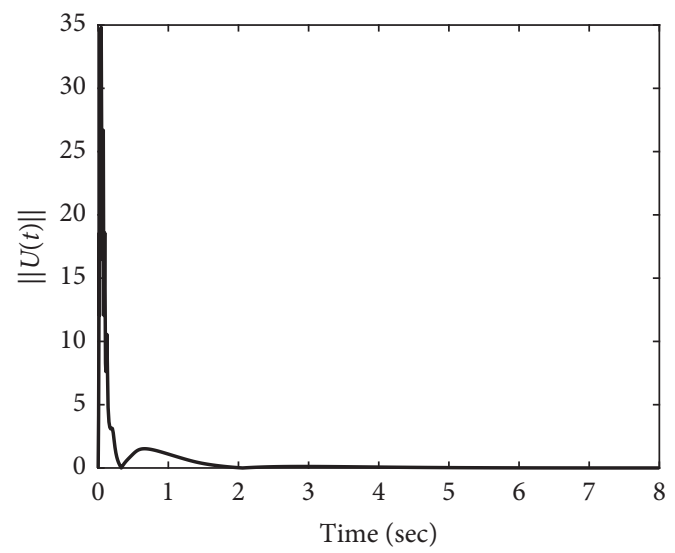

FIGURE 6: The control input response with the proposed continuous control input.

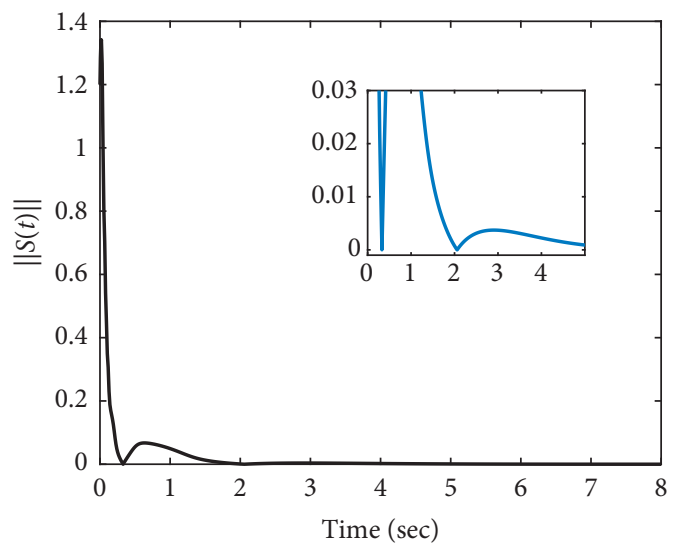

FIgURE 7: The switching function response with the continuous control input.

The initial state condition is given by $x_{0}=$ $\left[\begin{array}{lll}0 & -1.5 & 0.67\end{array}\right]^{T}$. The controller parameters are given by $j=8, \delta=8, \alpha=3$, and $\widehat{\eta}_{0}=0$. Let $K=\left[\begin{array}{ccc}-34.3 & 7 & 0 \\ 0 & 0 & 0.6667\end{array}\right]$, 


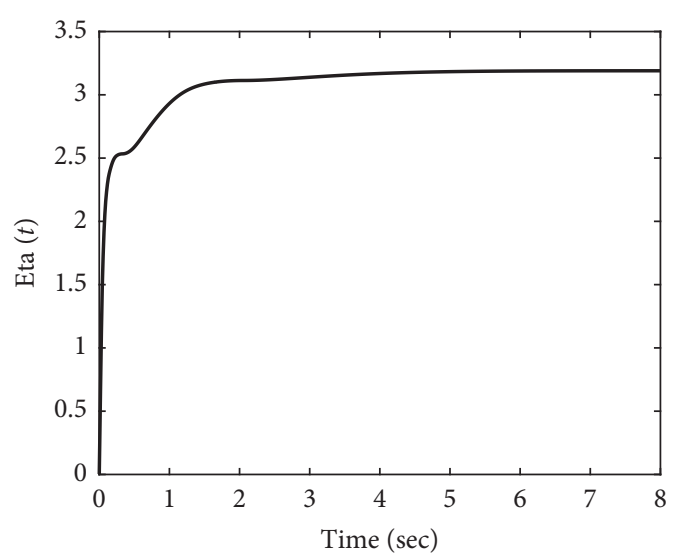

FIgURE 8: The time response for the adaptive parameter with the proposed continuous controller.

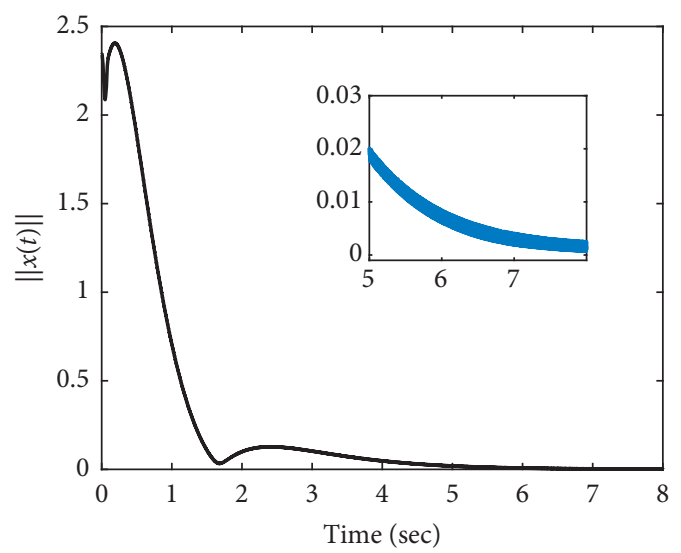

FIgURE 9: The system state response with the traditional SMC controller.

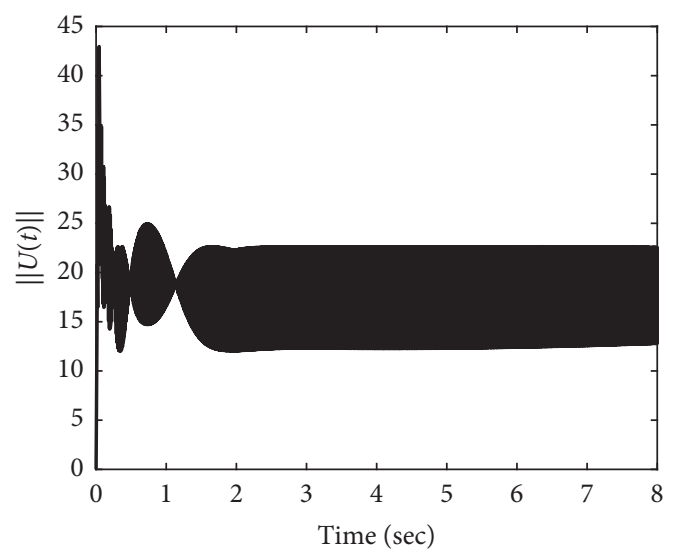

FIGURE 10: The control input response with the traditional SMC controller.

and the eigenvalues of the system are placed on $\{-2,-3,-1\}$. Also, the nonlinear function is defined as

$$
\varphi(u(t))=\left(1+0.3 \sin \left(u_{2}(t)\right)\right) u(t) .
$$

Under the input nonlinearity, the corresponding state responses, the proposed controller (13), the sliding surface

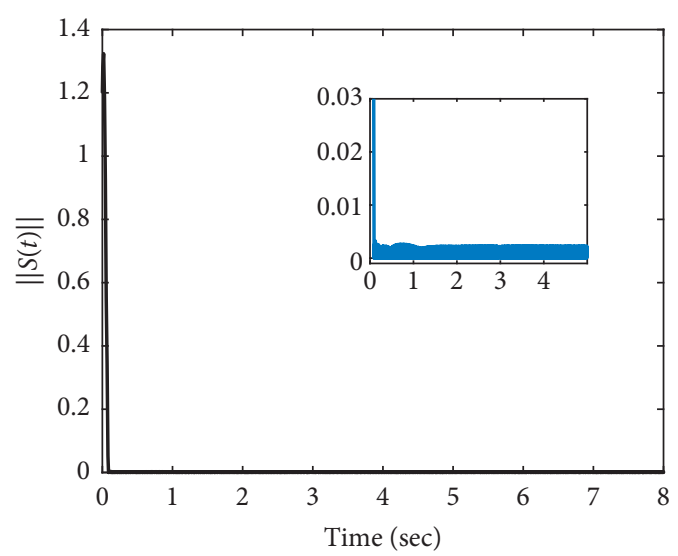

FIGURE 11: The switching function response with the traditional SMC controller.

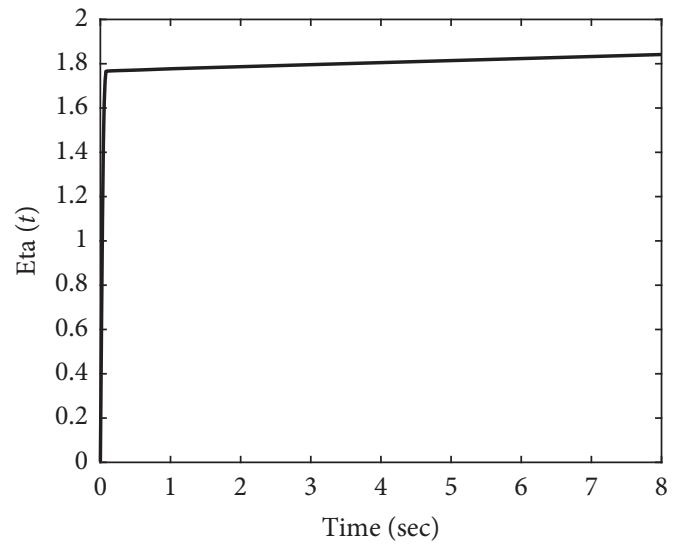

FIGURE 12: The time response for the adaptive parameter with the traditional SMC controller.

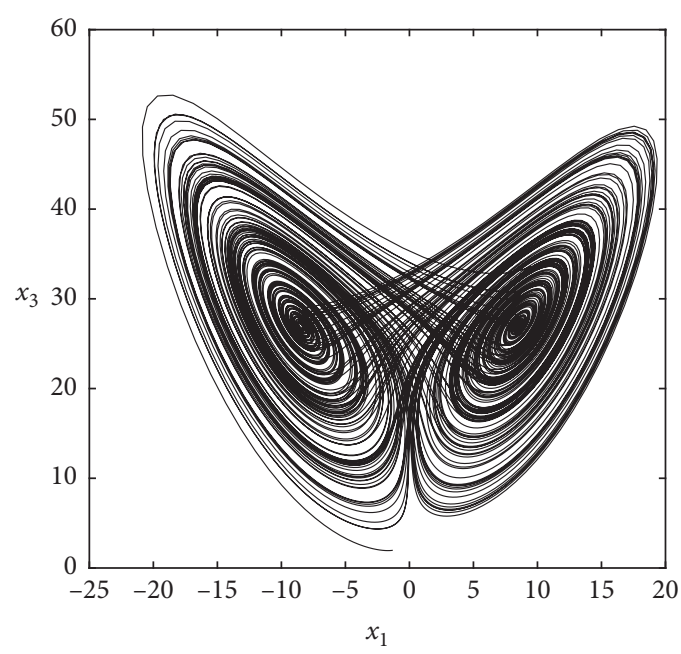

FIGURE 13: The chaos response of Lorenz system without control force.

(8), and adaptive parameter are shown in Figures 14-17, respectively. To compare the proposed approach with the traditional SMC, the tanh function is also replaced by the 


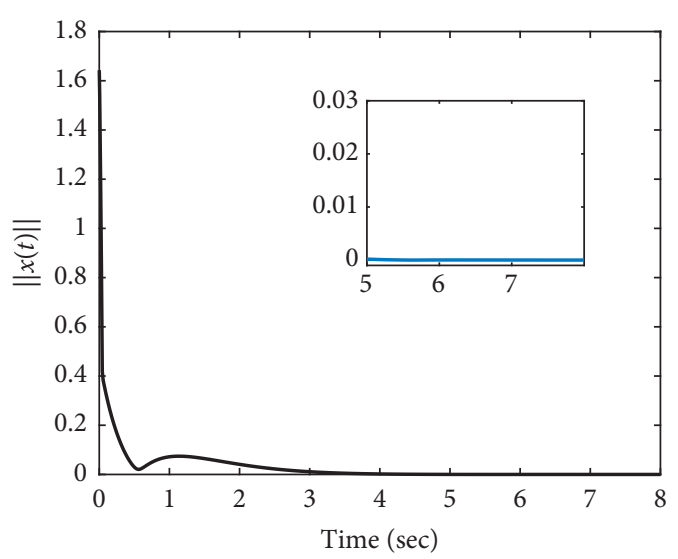

FIGURE 14: The system state response with the proposed continuous controller.

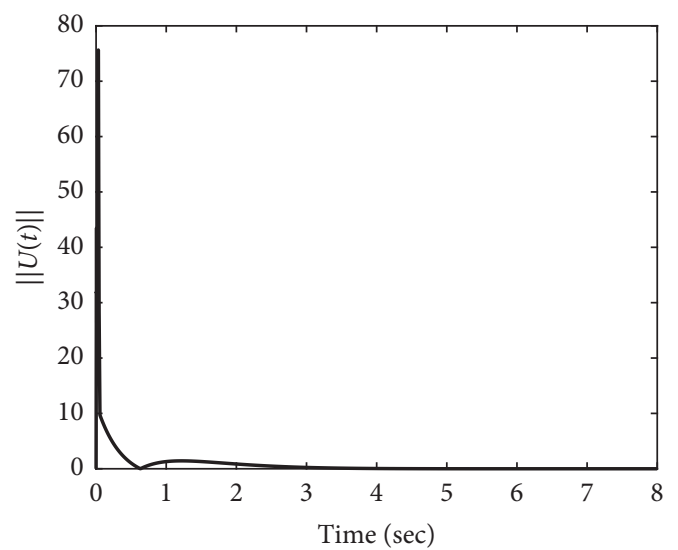

FIgURE 15: The control input response with the proposed continuous control input.

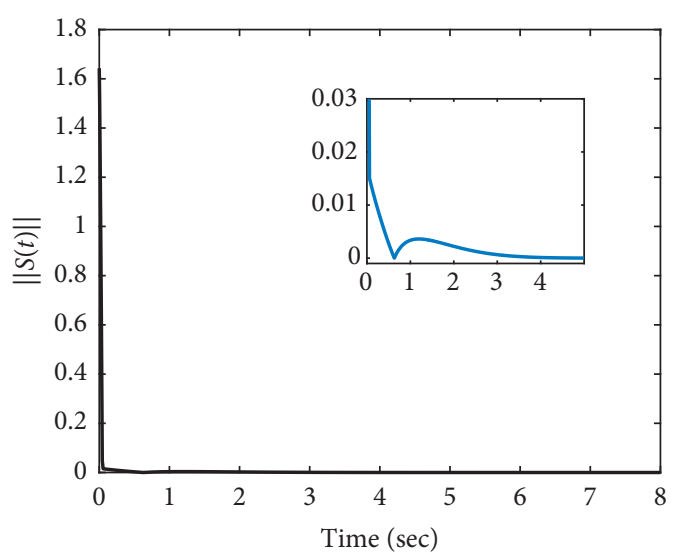

FIgURE 16: The switching function response with the proposed switching function.

traditional sign function in the controller (13). Based on traditional SMC, the corresponding state responses, the control input, the sliding surface, and adaptive parameter are shown in Figures 18-21. Comparing Figures 14-17 with Figures 18-21 shows that the undesired chattering

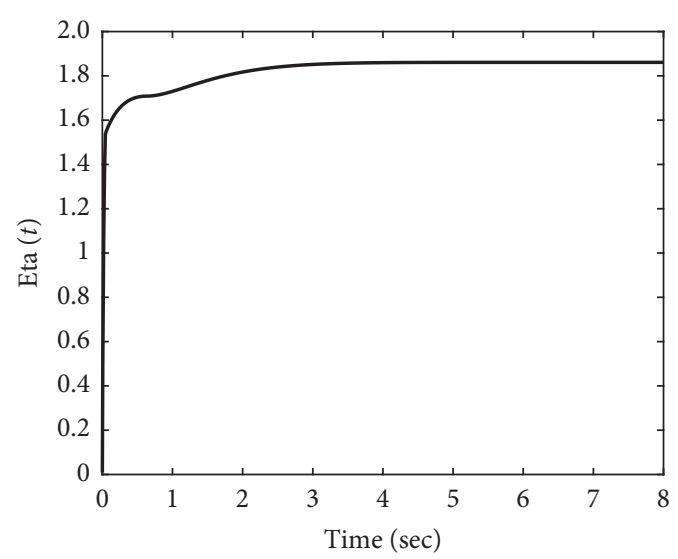

Figure 17: The time response of Example 2 for the adaptive parameter with the proposed continuous controller.

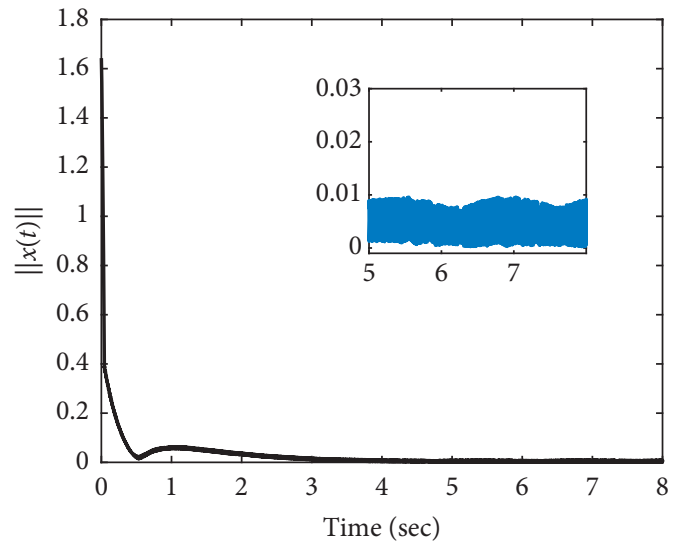

FIgURE 18: The system state response with the traditional SMC controller.

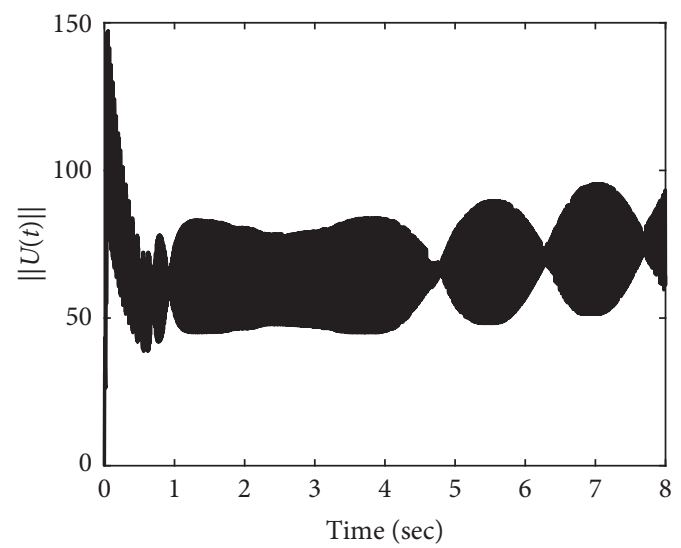

FIgURE 19: The control input response with the traditional SMC controller.

phenomenon is suppressed by using the proposed control law (13); hence, the results demonstrate the validity of proposed method.

From the simulation results above, it is concluded that the proposed method is effective and the chattering can be eliminated due to the adaptive continuous SMC even when 


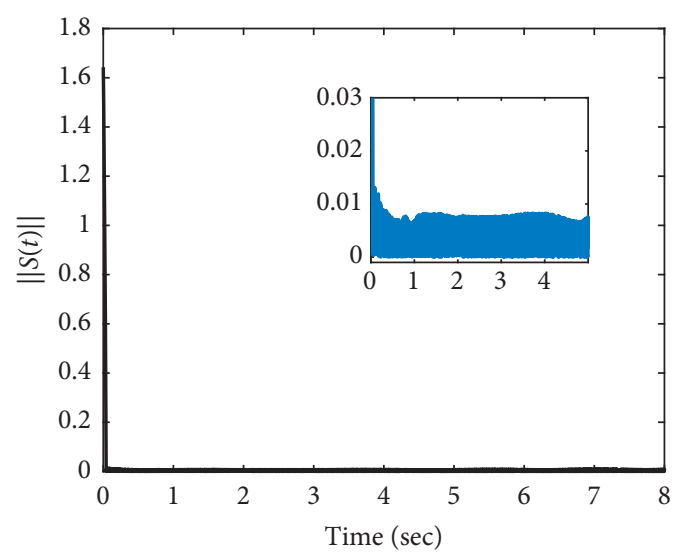

FIGURE 20: The switching function response with the traditional SMC controller.

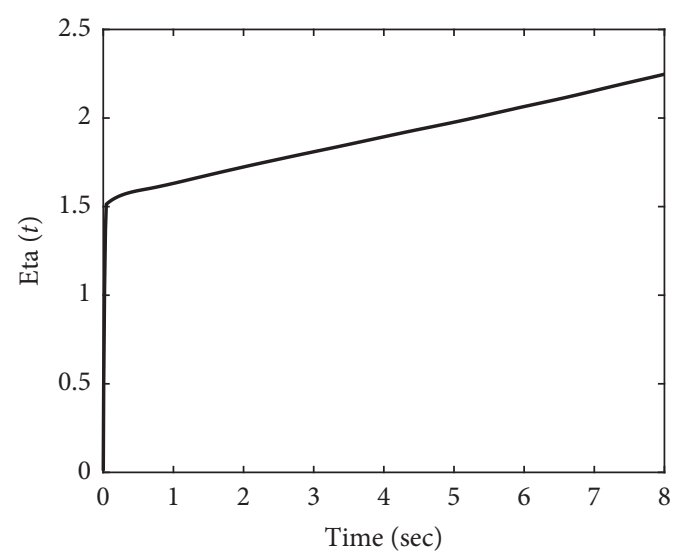

FIGURE 21: The time response for the adaptive parameter with the traditional SMC controller.

the controlled systems are subjected to unknown input nonlinearities.

\section{Conclusions}

This paper has proposed a continuous adaptive SMC design for chaos suppression of a general class of chaotic systems. In contrast to the previous works, the type of continuous adaptive SMC with smooth hyperbolic tangent function is newly introduced such that not only the chaos of systems can be suppressed but also the chattering in conventional SMC can be eliminated even with unknown input nonlinearity. Numerical simulations have verified the effectiveness of the proposed method.

\section{Data Availability}

The simulation data used to support the findings of this study are included within the article.

\section{Conflicts of Interest}

The authors declare that there are no conflicts of interest regarding the publication of this paper.

\section{Acknowledgments}

This study was supported by the Ministry of Science and Technology of R.O.C (MOST-108-2221-E-006-213-MY3 and MOST-107-2221-E-366-002-MY2).

\section{References}

[1] X. Liu and Y. Han, "Finite time control for MIMO nonlinear system based on higher-order sliding mode," ISA Transactions, vol. 53, no. 6, pp. 1838-1846, 2014.

[2] M. Singla, L.-S. Shieh, G. Song, L. Xie, and Y. Zhang, "A new optimal sliding mode controller design using scalar sign function," ISA Transactions, vol. 53, no. 2, pp. 267-279, 2014.

[3] M. Rahmani and M. H. Rahman, "An upper-limb exoskeleton robot control using a novel fast fuzzy sliding mode control," Journal of Intelligent \& Fuzzy Systems, vol. 36, no. 3, pp. 2581-2592, 2019.

[4] M. Rahmani, M. H. Rahman, and J. Ghommam, "A 7-DoF upper limb exoskeleton robot control using a new robust hybrid controller," International Journal of Control, Automation and Systems, vol. 17, no. 4, pp. 986-994, 2019.

[5] G. Liu, L. Zhang, and X. Guan, "Cooperative stabilization for linear switched systems with asynchronous switching," IEEE Transactions on Systems Man Cybernetics-Systems, vol. 49, no. 6, pp. 1081-1087, 2019.

[6] X. Chen and T. Fukuda, "Robust adaptive quasi-sliding mode controller for discrete-time systems," Systems \& Control Letters, vol. 35, no. 3, pp. 165-173, 1998.

[7] C.-F. Huang, T.-L. Liao, C.-Y. Chen, and J.-J. Yan, “The design of quasi-sliding mode control for a permanent magnet synchronous motor with unmatched uncertainties," Computers and Mathematics with Applications, vol. 64, no. 5, pp. 10361043, 2012.

[8] M.-C. Pai, "Discrete-time output feedback quasi-sliding mode control for robust tracking and model following of uncertain systems," Journal of the Franklin Institute, vol. 351, no. 5, pp. 2623-2639, 2014.

[9] A. H. Nayfeh and B. Balachandran, Applied Nonlinear Dynamics, John Wiley \& Sons Inc, New York, NJ, USA, 1995.

[10] E. Ott, C. Grebogi, and J. A. Yorke, "Controlling chaos," Physical Review Letters, vol. 64, no. 11, pp. 1196-1199, 1990.

[11] H.-T. Yau and J.-J. Yan, "Robust controlling hyperchaos of the Rössler system subject to input nonlinearities by using sliding mode control," Chaos, Solitons \& Fractals, vol. 33, no. 5, pp. 1767-1776, 2007.

[12] H.-T. Yau and J.-J. Yan, "Chaos synchronization of different chaotic systems subjected to input nonlinearity," Applied Mathematics and Computation, vol. 197, no. 2, pp. 775-788, 2008.

[13] G. Chen, "A simple adaptive feedback control method for chaos and hyper-chaos control," Applied Mathematics and Computation, vol. 217, no. 17, pp. 7258-7264, 2011.

[14] G. Chen, "Controlling chaotic and hyperchaotic systems via a simple adaptive feedback controller," Computers and Mathematics with Applications, vol. 61, no. 8, pp. 2031-2034, 2011.

[15] G. B. Maganti and S. N. Singh, "Output feedback form of Chua's circuit and modular adaptive control of chaos using single measurement," Chaos, Solitons and Fractals, vol. 28, no. 3, pp. 724-738, 2006.

[16] H. Salarieh and A. Alasty, "Adaptive chaos synchronization in Chua's systems with noisy parameters," Mathematics and Computers in Simulation, vol. 79, no. 3, pp. 233-241, 2008. 
[17] J. M. Nazzal and A. N. Natsheh, "Chaos control using slidingmode theory," Chaos, Solitons and Fractals, vol. 33, no. 2, pp. 695-702, 2007.

[18] H. Salarieh and A. Alasty, "Control of stochastic chaos using sliding mode method," Journal of Computational and Applied Mathematics, vol. 225, no. 1, pp. 135-145, 2009.

[19] A. Alfi, "Chaos suppression on a class of uncertain nonlinear chaotic systems using an optimal $\mathrm{H}_{\infty}$ adaptive PID controller," Chaos, Solitons \& Fractals, vol. 45, no. 3, pp. 351-357, 2012.

[20] S. Das, A. Acharya, and I. Pan, "Simulation studies on the design of optimum PID controllers to suppress chaotic oscillations in a family of Lorenz-like multi-wing attractors," Mathematics and Computers in Simulation, vol. 100, pp. 7287, 2014.

[21] D. Davendra, I. Zelinka, and R. Senkerik, "Chaos driven evolutionary algorithms for the task of PID control," Computers \& Mathematics with Applications, vol. 60, no. 4, pp. 1088-1104, 2010.

[22] E. G. Awad and R. Yassen, "Chaos and optimal control of a coupled dynamo with different time horizons," Chaos, Solitons and Fractals, vol. 41, no. 2, pp. 698-710, 2009.

[23] F. R. Chavarette, J. M. Balthazar, J. L. P. Felix, and M. Rafikov, "A reducing of a chaotic movement to a periodic orbit, of a micro-electro-mechanical system, by using an optimal linear control design," Communications in Nonlinear Science and Numerical Simulation, vol. 14, no. 5, pp. 1844-1853, 2009.

[24] C.-C. Fuh, "Optimal control of chaotic systems with input saturation using an input-state linearization scheme," Communications in Nonlinear Science and Numerical Simulation, vol. 14, no. 8, pp. 3424-3431, 2009.

[25] Y. Miladi, M. Feki, and N. Derbel, "Stabilizing the unstable periodic orbits of a hybrid chaotic system using optimal control," Communications in Nonlinear Science and Numerical Simulation, vol. 20, no. 3, pp. 1043-1056, 2015.

[26] M. T. Yassen, "The optimal control of Chen chaotic dynamical system," Applied Mathematics and Computation, vol. 131, no. 1, pp. 171-180, 2002.

[27] C. Hua and X. Guan, "Robust control of time-delay chaotic systems," Physics Letters A, vol. 314, no. 1-2, pp. 72-80, 2003.

[28] S. Nguang and P. Shi, "Robust $\mathrm{H}_{\infty}$ output feedback control design for fuzzy dynamic systems with quadratic D stability constraints: an LMI approach," Information Sciences, vol. 176, no. 15, pp. 2161-2191, 2006.

[29] M. Zhao and J. Wang, " $\mathrm{H}_{\infty}$ control of a chaotic finance system in the presence of external disturbance and input time-delay," Applied Mathematics and Computation, vol. 233, pp. 320-327, 2014.

[30] F.-H. Hsiao, "Robust $\mathrm{H}_{\infty}$ fuzzy control of dithered chaotic systems," Neurocomputing, vol. 99, pp. 509-520, 2013.

[31] Y.-F. Peng, "Robust intelligent sliding model control using recurrent cerebellar model articulation controller for uncertain nonlinear chaotic systems," Chaos, Solitons and Fractals, vol. 39, no. 1, pp. 150-167, 2009.

[32] L. Gao, D. Wang, and Y. Wu, "Non-fragile observer-based sliding mode control for Markovian jump systems with mixed mode-dependent time delays and input nonlinearity," Applied Mathematics and Computation, vol. 229, pp. 374-395, 2014.

[33] J. Li, W. Li, and Q. Li, "Sliding mode control for uncertain chaotic systems with input nonlinearity," Communications in Nonlinear Science and Numerical Simulation, vol. 17, no. 1, pp. 341-348, 2012.

[34] Q. Hu, G. Ma, and L. Xie, "Robust and adaptive variable structure output feedback control of uncertain systems with input nonlinearity," Automatica, vol. 44, no. 2, pp. 552-559, 2008.

[35] M. T. Yassen, "Adaptive control and synchronization of a modified Chua's circuit system," Applied Mathematics and Computation, vol. 135, no. 1, pp. 113-128, 2003.

[36] C. Li and J. C. Sprott, "Multistability in Lorenz system: a broken butterfly," Int. International Journal of Bifurcation and Chaos, vol. 24, no. 10, 2014. 


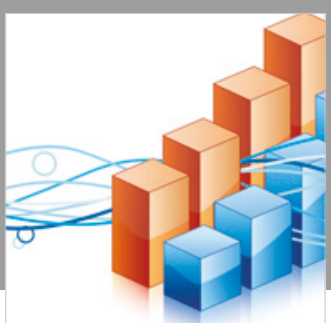

Advances in

Operations Research

\section{-n-m}
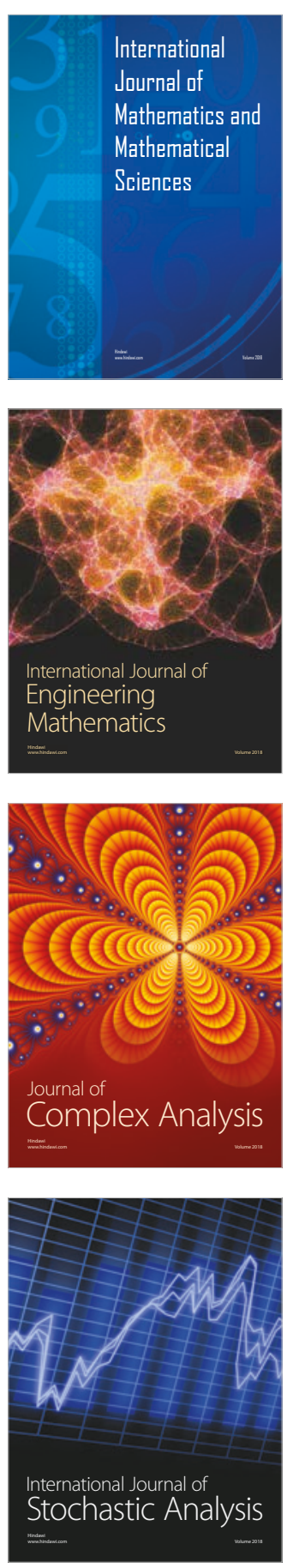
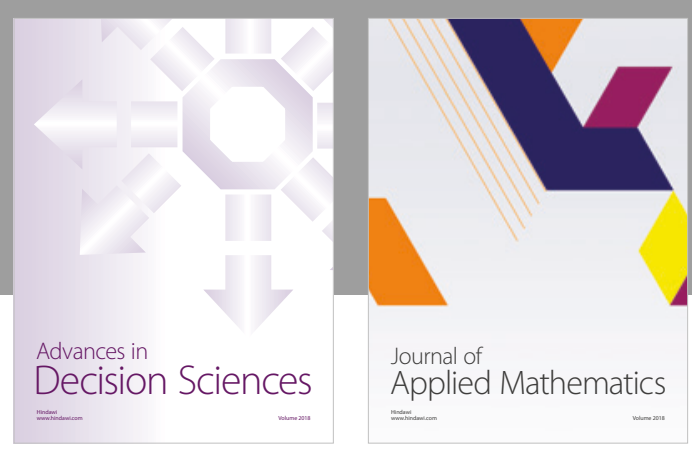

Journal of

Applied Mathematics
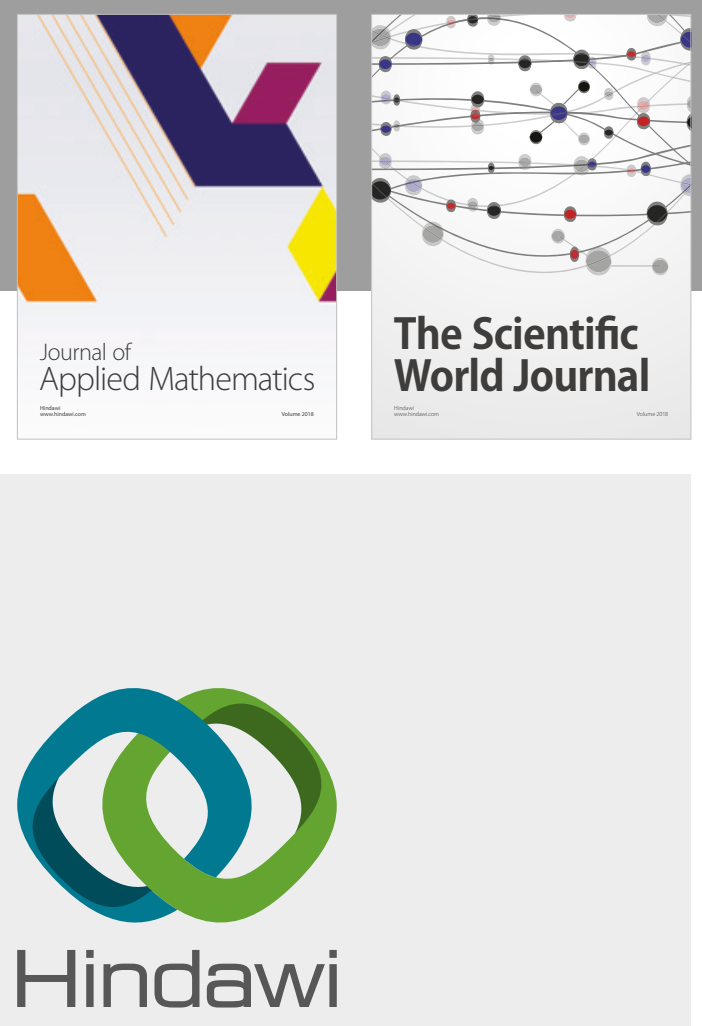

Submit your manuscripts at

www.hindawi.com

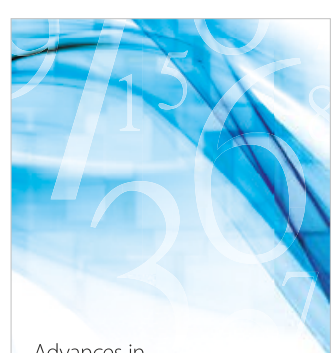

Advances in
Numerical Analysis
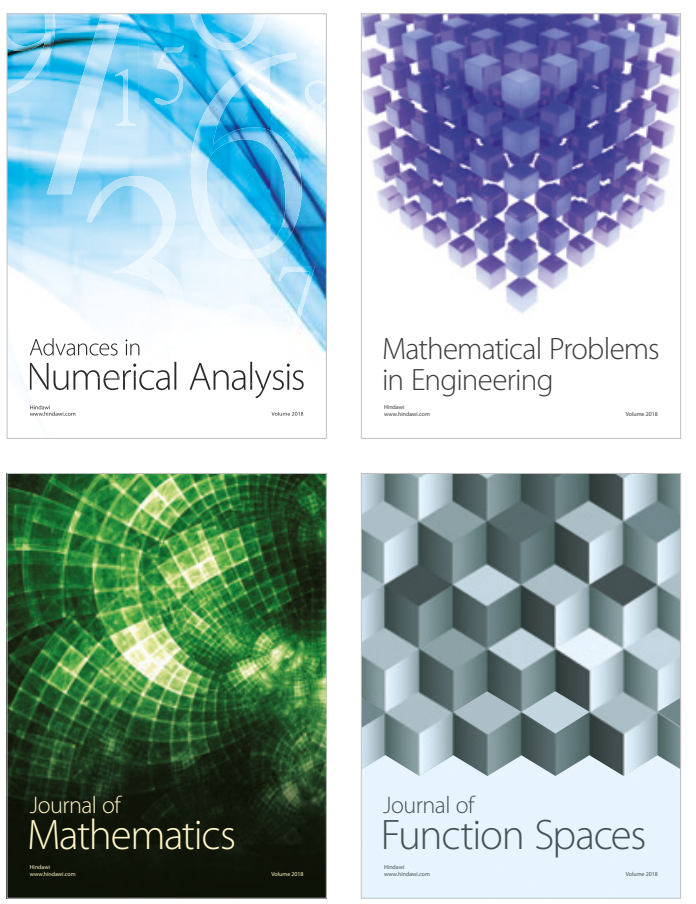

Mathematical Problems in Engineering

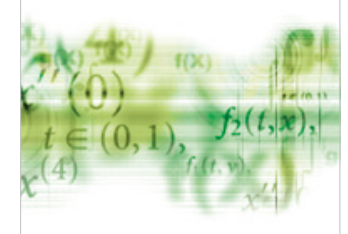

International Journal of

Differential Equations

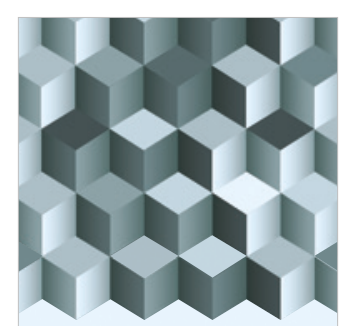

Journal of

Function Spaces

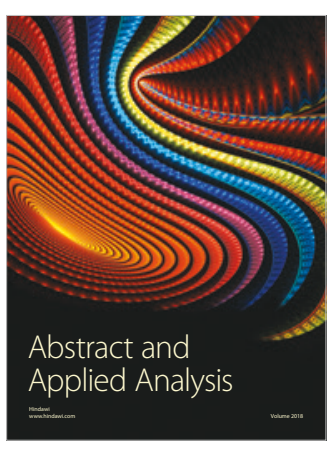

The Scientific

World Journal

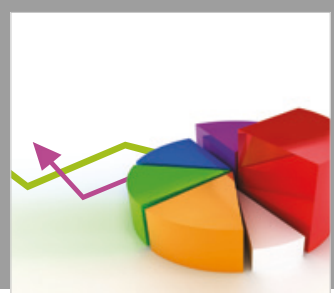

Journal of

Probability and Statistics
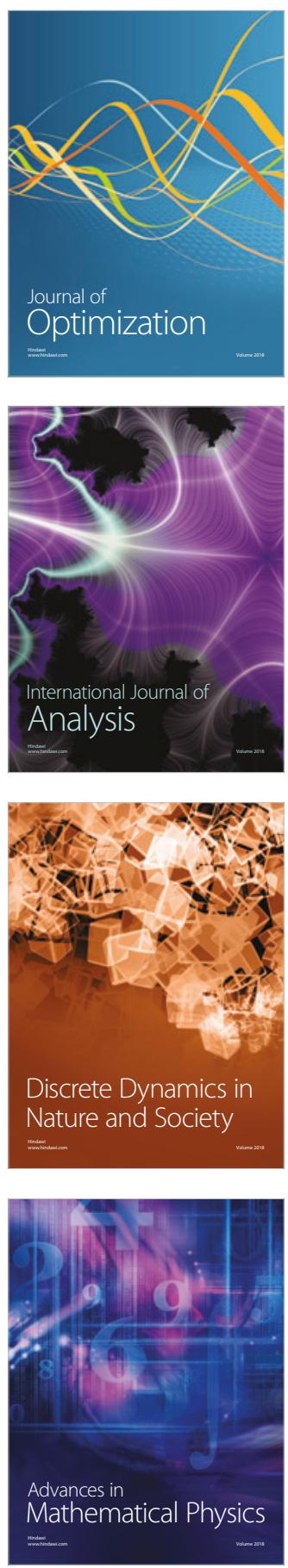